\title{
CZU: 633.111:575 \\ https://doi.org/10.53040/gppb7.2021.40 \\ EPIGENETIC IN HERITANCE AND SELECTION OF HEAT AND FROST RESISTANT WHEAT (Triticum aestivum L.) GENOTYPES
}

\author{
Jelev Natalia ${ }^{1}, Z_{\text {dioruc Nina }}{ }^{1}$, Ralea $T^{1}$., Dascaliuc Al. ${ }^{1}$, Parii Iaroslav $^{2}$, Parii Iulia ${ }^{2}$ \\ ${ }^{1}$ Institute of Genetics, Physiology and Plant Protection, Chisinau, Republic of Moldova \\ ${ }^{2}$ All Ukrainian Institute of Plant Breeding, Kiev, Ukraine \\ e-mail: natalia.jelev@igfpp.md
}

\begin{abstract}
The primary resistance and plasticity of the response to shock with negative temperatures (SNT) or heat shock (HS) of different winter wheat genotypes grown in Ukraine and then reproduced in Moldova are determined. The values of the mentioned parameters specifically varied depended on the genotype specificity and conditions of seeds reproduction. Thus, data support the hypothesis about the specificity of trans-generational inheritance of wheat genotypes adaptations to extreme temperatures. Furthermore, the correlation coefficient between the resistance SNT value to HS and inversed value (HS/SNT) ratio may indicate the epigenetically inherited effects.
\end{abstract}

Key words: winter wheat, heat shock, sock with negative temperature, plasticity

\section{Introduction}

Due to progress in selection and plants cultivation technologies, crops with high productivity have been obtained over the centuries. At the same time, appreciating the quality of agricultural products, the emphasis was increasingly accepted on the users' taste preferences, directed and formed by advertisement, aimed to increase companies' profits without considering the population health. For maximizing profit, new technologies involve creating specific cultivation conditions that ensure the reduction/exclusion of competition between cultivated genotypes and spontaneous plants, the influence of biotic and abiotic stressors on plants. As a result, the development of agriculture has been continuously directed towards increasing the economic profit of new products and technologies compared to the previous ones. However, in the background remained such vital issues as protection of the environment, vigor, viability, and compliance of the properties of selected genotypes to environmental conditions long-term effects of agricultural products on human health. Thus, the continuous amplification of the damages caused to the environment and the population's health due to implementing this strategy becomes more evident. Therefore, more efforts are needed to increase the quality and quantity of agricultural products made by natural means qualified as organic. As a result, the increase in organic agriculture productivity is slower than in traditional but continuous progress in time. In the long run, due to avoiding the continuously increasing risks caused by conventional technologies, organic farming results are superior to traditional ones. During evolution, plants developed different adaptations to environmental conditions that spread over the Earth's entire surface. Knowledge and consideration of these mechanisms is an important prerequisite for the successful promotion of organic farming.

The mechanisms of plants' adaptation to environmental conditions can be divided into two groups: the mechanisms that ensure the reduction/avoidance of the action of stressors on plants; and those of functional elimination (reparation) of the damages caused by stress $[1 ; 2]$. Traditionally is considered that the specifics of all adaptation mechanisms are determined genetically. Still, more and more data currently demonstrate the transmission of acquired traits in the ontogenesis of plants to future generations, involving hereditary epigenetic mechanisms [3; 4]. The transgenerational information transmission has been multilaterally investigated with several plant species, including flax genotrophs [4] and the inheritance of vernalization and photoperiod requirements in wheat [5]. Research on the possibility of "transforming" the winter wheat into spring wheat and vice versa marked Soviet biological science in the middle of the twentieth century. Considering that currently, the transmission between cells and plant organs of the traits acquired in ontogenesis is generally recognized, we focused on the inheritance of the primary resistance to thermal stress by seeds of wheat genotypes multiplied under different environmental conditions. We mention that the primary stress resistance is a fundamental characteristic determined by the resistance of organisms to stressors before the induction of adaptation processes, which take place under their influence during ontogenesis $[1,2,6]$. 


\section{Materials and methods}

We used in the studies seeds of 10 varieties, and 40 lines of hexaploid wheat multiplied in the Kharkiv region of Ukraine (2015-2016) and the Chisinau area of Moldova (2016-2017). The seeds of different wheat genotypes were shocked by SNT or HS and then determined their influence on the germination percentage [7]. The average rate of seeds germination, standard deviation, and the mathematical expectation of the mean of the response of wheat genotypes to SNT or HS, was determined [8].

\section{Results and discussions}

Initially, in the analysis were used the seeds of 50 wheat genotypes multiplied in Ukraine. We characterized the plasticity of genotype response to extreme temperatures based on their primary resistance to high temperatures and negative ones. Therefore, we have considered that the integral characterization of the plasticity of genotype reaction to extreme temperatures can be the appreciation of the summary value of the percentages of seeds germinated after separate exposure to SNT and HS. Therefore, the genotypes characterized by the sum value less than $100 \%$, between $100 \%$ and $140 \%$, and higher than $140 \%$ were considered genotypes with a low, moderate, and high level of plasticity, respectively. Based on the obtained results, 20, 18, and 12 from the 50 genotypes were characterized with low, moderate, and high levels of plasticity, respectively. Furthermore, the correlation coefficient between the percentage of germinated after exposure to SNT and HS of the 50 wheat genotypes seeds was negative and equal to -0.4632 . Therefore, we must highlight that all genotypes from the group with high plasticity also showed increased resistance to SNT and HS.

Simultaneously, only some genotypes with medium or low plasticity demonstrated high resistance to SNT or HS. Within the groups with low, moderate, and high plasticity, the correlation coefficient between the values that characterize the plasticity and the percentage of seeds that germinated after exposure to SNT or HS was positive, ranging between 0.3861 and 0.6568 . At the same time, for the mentioned groups of genotypes, the correlation coefficient between the percentage of seeds that germinated after exposure to SNT and those exposed to HS was negative, being respectively equal to $-0.6460,-0.6291$, and -0.2076 . Thus, these data confirm the negative correlation between the plants' resistance to high temperatures and frost [2].

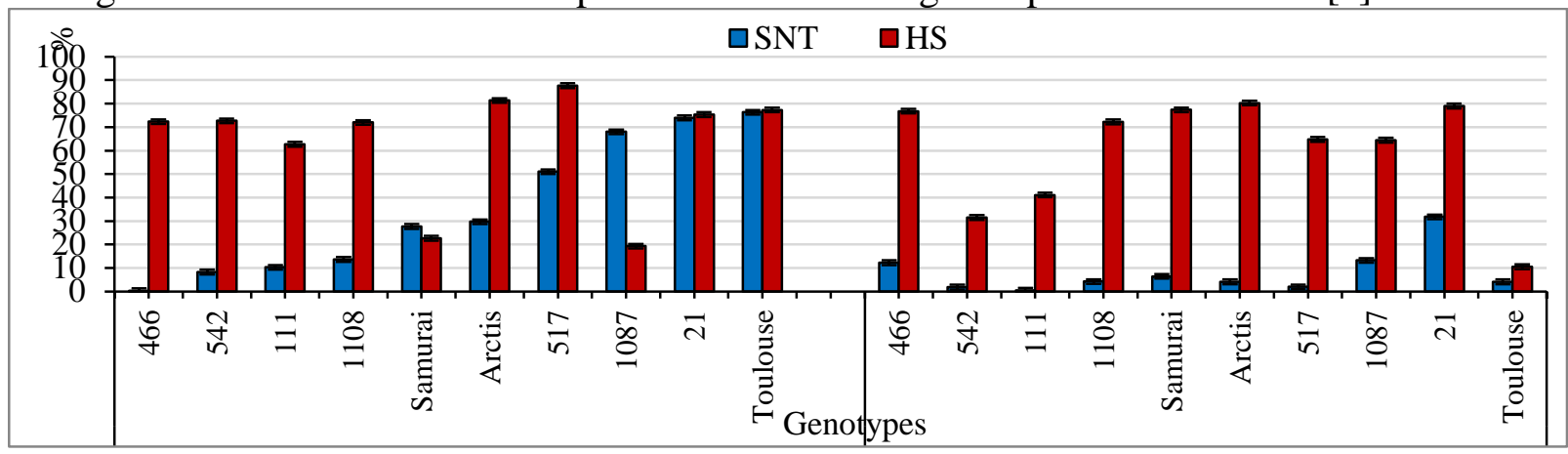

Figure. The percentage of seeds of the ten winter wheat genotypes germinated after exposure for 8 hours to SNT at $-7^{\circ} \mathrm{C}$ (blue). The percentage of germinated after exposure for 30 minutes to $\mathrm{HS}$ at $50^{\circ} \mathrm{C}$ (red). On the left part of the figure are presented the data obtained with seeds multiplied in 2015-2016 in the Kharkiv region (Ukraine) winter wheat lines and varieties plasticity, and on the right - the data obtained with the seeds multiplied in 2016-2017 in the Chisinau area (R.Moldova).

We mention that in our research, seeds were exposed to doses of SNT or HS that caused a $50 \%$ decrease in the germination of genotypes with moderate resistance to these doses of stressors. Therefore, we assumed that the deviation of the value of the percentage of germination by hocks with extreme temperatures from this value $(50 \%)$ could characterize the plasticity of the response of genotypes to extreme temperatures. Consequently, we calculated the correlation coefficient between the values of the ratio between the percentage of seeds germinated after exposure to SNT to that of those geminated after exposure to HS (SNT/HS), on the one hand, and the values of the inversed ratio (HS/SNT) for the groups separated according to the level of genotypes plasticity.

On the other hand, for the group of genotypes with high plasticity, the expected values of the correlation coefficient between mentioned two ratios for the group with high plasticity are expected to be positive. Indeed, in our experiments, the correlation coefficient between these ratios for groups with low, moderate and high plasticity reached the values $-0.4448,-0.9283$, and 0.9971 , respectively. In this way, the correlation coefficient is positive (high positive) only for the group of genotypes with high plasticity. 
The following figure presents the data on the primary summary resistance (plasticity) to SNT and HS of the seeds of 10 wheat genotypes multiplied in Ukraine or Moldova. They show that after cultivation in Ukraine, the seeds of genotypes 466, 542, 111, 1108, Samurai.

Arctis, were characterized with low resistance; those of the genotypes 517 and 1087 - with medium resistance, and seeds of genotypes 21 and Toulouse - showed high resistance to SNT action. After being cultivated in R.Moldova, seeds of all genotypes, except those of the variety Toulouse with medium resistance to SNT, have shown low resistance to SNT. In contrast, except for seeds of lines 21, 542, and 111 with a low resistance to HS, the multiplied in Moldova seeds of the other seven genotypes showed medium or high resistance to HS.

Simultaneously, the seeds of lines 466 and 1087, varieties Samurai and Toulouse, obtained from the plants grown in R.Moldova, showed relatively high resistance to HS and the correlation coefficient of genotypes resistance to HS and SNT reached the value of 0.4340 . We mention that the correlation coefficient of the resistance to SNT of the seeds obtained from the plants cultivated in Moldova and Ukraine was equal to 0.4810 when the respective correlation of the seed's resistance to HS was negative, equal to -0.314 . The correlation coefficients of resistance to SNT of seeds multiplied in Ukraine and Moldova were positive. This value suggests that, although, in general, the resistance to SNT of seeds reproduced in Moldova was lower compared to those reproduced in Ukraine, the differences between genotypes tended to be in the same direction (to decrease the resistance to SNT) for the majority of genotypes. The negative correlation coefficient between the HS resistance of the seeds propagated in Ukraine and Moldova demonstrates the opposite direction of changing the HS resistance of seeds obtained from plants grown in Moldova compared to those of the seeds multiplied in Ukraine. For example, the HS resistance of seeds of the lines 542, 111, and the variety Toulouse was higher in seeds obtained from plants grown in Ukraine, when the seed resistance of varieties Samurai, Arctis, and line 1087, on the contrary, was higher in the case of their multiplication in Ukraine.

\section{Conclusions}

1. The primary resistance seeds of different wheat genotypes' to the application of the HS and SNT differ and, at the same time, are influenced by the environmental conditions of their reproduction.

2. The obtained data support the hypothesis of epigenetic inheritance of some ontogenetically acquired traits.

Research was carried out within the project of the State Program 20.80009.7007.07 "Determining the parameters that characterize the resistance of plants with the different level of organization to the action of extreme temperatures in order to reduce the effects of climate change", financed by the National Agency for Research and Development.

\section{Bibliography}

1. DASCALIUC, A., IVANOVA, R., ARPENTIN, Gh. Systemic approach in determining the role of bioactive compounds. In Pierce, G.N.; Mizin V. I.; Omelcienko A., eds. Advanced Bioactive Compounds Countering the Effects of Radiological, Chemical, and Biological Agents, Strategies to counter biological damage; Series: NATO Science for Peace and Security Series A: Chemistry and Biology. Springer; 2013. p. 121-131. ISBN: 9789400765320

2. LEVITT, J. Chilling, Freezing and High Temperature Stresses. 2nd Edition. Academic Press; 1980. 510 p. ISBN: 9780323161633

3. MOZGOVA, I., MIKULSKI, P., PECINKA, A. et al. Epigenetic Mechanisms of Abiotic Stress Response and Memory in Plants. In Alvarez-Venegas R., De-la-Peña C., Casas-Mollano .JA. editors Epigenetics in Plants of Agronomic Importance: Fundamentals and Applications. Transcriptional Regulation and Chromatin. Remodelling in Plants. Switzerland: Springer; 2019. p. 1-64. DOI: org/10.1007/978-3-030-14760-0

4. CULLIS, C. A. Origin and Induction of the Flax Genotrophs. In: Cullis C. (eds) Genetics and Genomics of Linum. Plant Genetics and Genomics: Crops and Models, Springer, Cham 2019. V.23. p. 227-234. DOI: org/10.1007/978-3-030-23964-0_14

5. DASCALIUC, A. Yarovizatsiya: itogi problemy perspektivy. Kishinau, Shtiintsa, 1993, 151 p. ISBN 5-37601689-7 (In Russian)

6. DASCALIUC, A., ZDIORUC, N., RALEA, T. Determination of Triticum aestivum L. primary resistance to high temperature. Plant Physiology and Genetics. 2021;53:336-345. DOI: org/10.15407/frg2021.04.336

7. JELEV, N., DASCALIUC, A., PALEA, T., 3DIORUK, N., OBOZNÂI, A., PARII, IU., PARII, Ia. Evaluation of wheat (Triticum aestivum L.) genotypes primary comparative resistance to positive and negative temperatures. Buletinul Academiei de Ştiinţe a Moldovei, Ştiinţele vieţii. 2018, nr. 1(334), p.61-70. ISSN 1857-064X

8. CLEWER, A.G, SCARISBRICK, D.H. Practical statistics and experimental design for plant crop science. Chichester, New York: John Wiley \& Sons, LTD. 2001, 346 p. ISBN: 978-1-118-68566-2 